\title{
BMJ Open Use of the kidney failure risk equation to inform clinical care of patients with chronic kidney disease: a mixed- methods systematic review
}

\author{
Harjeet Kaur Bhachu, ${ }^{1,2,3}$ Anthony Fenton (1D , ${ }^{1,2}$ Paul Cockwell, ${ }^{1,4}$ \\ Olalekan Aiyegbusi, ${ }^{2,3,5,6}$ Derek Kyte (D) , ${ }^{2,3,7}$ Melanie Calvert (D) ${ }^{2,3,5,6}$
}

To cite: Bhachu HK, Fenton A, Cockwell $P$, et al. Use of the kidney failure risk equation to inform clinical care of patients with chronic kidney disease: a mixed-methods systematic review. BMJ Open 2022;12:e055572. doi:10.1136/ bmjopen-2021-055572

- Prepublication history and additional supplemental material for this paper are available online. To view these files, please visit the journal online (http://dx.doi.org/10.1136/ bmjopen-2021-055572).

Received 23 August 2021 Accepted 17 December 2021

Check for updates

(C) Author(s) (or their employer(s)) 2022. Re-use permitted under CC BY-NC. No commercial re-use. See rights and permissions. Published by BMJ.

For numbered affiliations see end of article.

Correspondence to

Dr Anthony Fenton;

a.fenton@bham.ac.uk

\section{ABSTRACT}

Rationale and objective The Kidney Failure Risk Equation (KFRE) predicts the risk of end-stage kidney disease in patients with chronic kidney disease (CKD). This study aimed to evaluate the impact of the utility of KFRE in clinical practice.

Study design Systematic review.

Setting and study populations Adult patients with CKD but not receiving renal replacement therapy enrolled in studies where KFRE was used in clinical care pathways.

Selection criteria for studies All studies published from April 2011 to October 2021 identified from Medline, Cumulative Index to Nursing and Allied Health Literature, Embase and reference and citation searches of included studies.

Data extraction Relevant data were extracted, and two reviewers independently assessed study quality using appropriate appraisal tools.

Analytical approach Findings reported as a narrative synthesis due to heterogeneity of the included studies. Results Of 1635 studies identified, 440 duplicates were removed. The remaining 1195 titles and abstracts were screened. All five studies for full-text review were included in the analysis. Three uses of KFRE were assessed: (1) primary to specialty care interface; (2) general nephrology to multidisciplinary care transition; and (3) treatment planning. Evidence of impact on number of patient referrals into nephrology care was conflicting. However, wait times improved in one study. Although KFRE identified high-risk patients for increased multidisciplinary support, there was concern patients stepped down, no longer meeting eligibility criteria, may lack access to services.

Conclusions This is the first systematic review of studies that have assessed the actual impact of KFRE in clinical practice with five studies of varying quality reported to date. Trials are in progress assessing the impact on clinical outcomes of using KFRE in clinical practice, and KFRE is being incorporated into guidelines for CKD management. Further studies are needed to assess the impact of KFRE on clinical care.

Trial registration number Protocol registered on PROSPERO before initiation of the study (Ref: CRD42020219926).
Strengths and limitations of this study

To the best of our knowledge, this is the first systematic review to examine the evidence for the impact of the use of the Kidney Failure Risk Equation in clinical practice.

- A mixed-methods approach was used to allow the inclusion of both quantitative and qualitative evidence.

- Study quality ranged widely, and some studies did not provide adequate detail of their population characteristics, such that generalisability was difficult to assess.

- Furthermore, the statistical analysis was limited in several studies.

\section{BACKGROUND}

The global prevalence of chronic kidney disease (CKD) is estimated to be $9.1 \%$, and CKD was the 12th leading cause of death in 2017. ${ }^{1}$ CKD is also associated with an increased risk of progression to end-stage kidney disease (ESKD). Risk stratification in CKD can enable more efficient care, with specialty care targeted to patients at higher risk of ESKD, while sparing those with low risk unnecessary intervention and undue anxiety associated with this. ${ }^{23}$

Most guideline criteria for referral of patients to specialist nephrology care use estimated glomerular filtration rate (eGFR), eGFR decline and urine albumin:creatinine ratio (ACR) thresholds rather than a quantified ESKD risk. ${ }^{4}$ Such criteria often result in the referral of patients at low risk of ESKD and the non-referral of patients at high risk. ${ }^{5}$ Therefore, recent CKD guidelines endorse risk-based thresholds for specialty referral and renal replacement therapy (RRT) planning. ${ }^{67}$

The best validated risk prediction model is the Kidney Failure Risk Equation (KFRE), 
which predicts the 2-year and 5-year risk of ESKD in patients with stages 3-5 CKD and has undergone extensive validation. ${ }^{8-10}$ The equation can easily be embedded into electronic medical records and is also readily available online. ${ }^{11}$

It is uncertain whether the use of the KFRE in clinical practice has a meaningful impact on clinical pathways and health outcomes and how patients and healthcare professionals view the KFRE. We carried out a systematic review of the available evidence of the impact of the use of the KFRE in clinical practice.

\section{METHODS}

The study protocol was registered on PROSPERO (Ref: CRD42020219926), and the study is reported as per the 'Preferred Reporting Items for Systematic Review and Meta-Analysis' (PRISMA) checklist. ${ }^{12}$

\section{Data sources and searches}

Two reviewers (HKB and AF) independently searched MEDLINE (Ovid), Cumulative Index to Nursing and Allied Health Literature (EBSCO) and Embase (Ovid) for studies between April 2011 to October 2021 that examined the impact of utilising the KFRE in patients with CKD.

The search strategies (online supplemental table S1) were developed with the support of an information specialist and used keywords, index terms and Medical Subject Headings terms tailored and applied to each individual database. No language restrictions were applied. We also hand searched the reference lists of included studies and performed a forward citation review of these studies and the original KFRE development study. ${ }^{13}$ All results obtained at each stage of the process were entered into EndNote V.X9.3.3 (Clarivate Analytics, Pennsylvania, USA), and duplicates were removed. Any disagreements regarding study inclusion were resolved by discussion or decided by a third reviewer (PC) where necessary.

\section{Study selection and eligibility criteria}

Studies were included if they:

1. Were published from April 2011 (the date of the initial KFRE paper publication) to October 2021.

2. Studied adults with CKD but not receiving renal replacement therapy (dialysis or kidney transplantation).

3. Used the KFRE to estimate the risk of ESKD.

4. Evaluated the actual rather than potential impact of using the KFRE in clinical practice.

Studies were excluded if they were development or validation studies only, narrative reviews, editorials, commentaries or opinions, or letters.

The two reviewers ( $\mathrm{HKB}$ and $\mathrm{AF}$ ) independently screened the titles and abstracts against the eligibility criteria. Potentially relevant studies were identified, and final inclusion was based on full-text examination. Reasons for exclusion following full-text review were documented.

\section{Data extraction and quality assessment}

The following key data were extracted: (1) study details (author, year of publication, title and location of study), (2) study type, (3) aim, (4) study population characteristics, (5) method, (6) results, (7) key findings, (8) strengths and limitations and (9) author conclusions.

Methodological validity was assessed independently by the two reviewers (HKB and $\mathrm{AF}$ ) using the Critical Appraisal Skills Programme appraisal tools, ${ }^{14}$ Joanna Briggs Institute Critical Appraisal Tools ${ }^{15}$ and Centre for Evidence-Based Management Critical Appraisal of a Survey. ${ }^{16}$

\section{Data synthesis and analysis}

Due to different study methods and the heterogeneity of study characteristics, individual analysis for quantitative studies and qualitative studies was not conducted. Findings were reported as a narrative synthesis adopting the methods presented by Popay et al ${ }^{17}$ : (1) theoretical reason for basis of evaluated intervention, that is, using the KFRE, (2) descriptive summary of study characteristics and critical appraisal, (3) exploration of associations within and between studies and (4) assessing the strength of evidence and limitations of the synthesis process.

\section{Patient and public involvement}

There was no patient and public involvement in the conduct of this systematic review.

\section{RESULTS}

The database search identified 1099 studies, and a further 536 studies were identified from the forward citation search of the KFRE development study. ${ }^{13}$ Four hundred and forty duplicates were removed, resulting in 1195 studies taken forward for the title and abstract screening. Five studies fulfilled the criteria for full-text review. No additional studies were identified from reference review and forward citation review of the selected studies. Results of the search are presented in the (PRISMA) flow $\operatorname{diagram}^{12}$ (figure 1).

\section{Study characteristics}

Table 1 presents the details of the included studies. Three studies took place in Canada, ${ }^{318} 19$ one in Australia ${ }^{20}$ and one in the USA. ${ }^{21}$ Four studies were quantitative, and one study used a mixed-methods approach, whereby the strengths of both qualitative (interviews) and quantitative (surveys) research elements were combined to gain a broader view of their experience applying the KFRE. ${ }^{3}$ The KFRE was used at three transition points: (1) the primary to specialty care interface, ${ }^{18}{ }^{20}$ (2) general nephrology to multidisciplinary care or advanced care kidney clinics ${ }^{319}$ and (3) to guide treatment planning in a private healthcare setting, ${ }^{21}$ including referrals to primary or nephrology care, medication changes and laboratory recommendations. No study stated the baseline risk used (North American or non-North American). Only two 


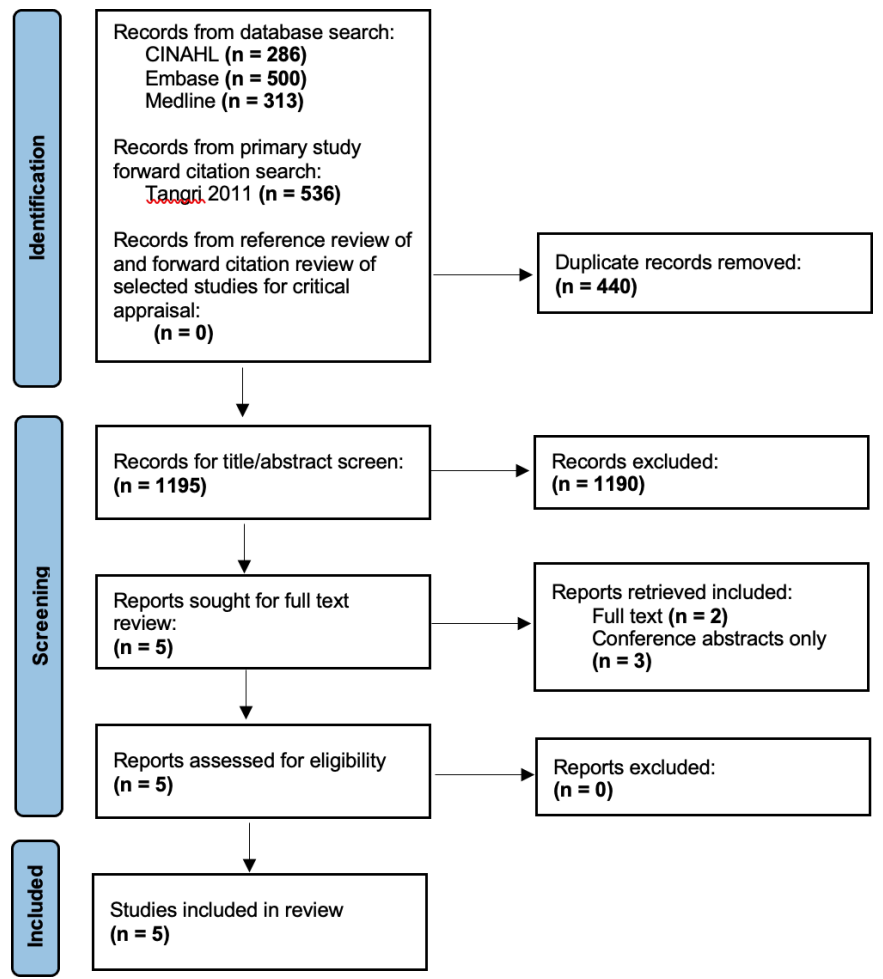

Figure 1 Flow diagram to show the studies identified from searches.

studies stated which version of the KFRE was used, but all studies stated if they calculated 2-year or 5-year ESKD risk.

Hingwala $e a^{18}$ applied a quasiexperimental (pre-post) study design to patients referred to three renal centres in the Manitoba Renal Program, Manitoba, Canada. Wait times (between referral and nephrology visit) and number of consults were compared from the periods preimplementation and postimplementation of the new triage criteria: significant criteria or 4-variable KFRE 5-year risk $>3 \%$. Patients with a 3\%-10\% risk were booked as non-urgent $(<6$ months) and those with a $>10 \%$ risk were booked as urgent $(<4$ weeks).

A similar pre-post study design was used in the study by Hong et $a l,{ }^{20}$ where they implemented new criteria, using a 5-year KFRE score of $>3 \%$, for patients referred to the St George Hospital Renal Department (New South Wales, Canada). Patients referred with a $<3 \%$ risk were accepted at the discretion of a nephrology consultant. The number of consults pretriage and post-triage periods were compared.

The KFRE was applied within a secondary care setting in two studies. Smekal et al used a mixed-methods approach to gauge the views of patients and providers 1 year after implementing the KFRE in 2017 to guide transition into the CKD multidisciplinary clinic. Criteria for transition were KFRE 2-year risk $\geq 10 \%$ or eGFR $\leq 15 \mathrm{~mL} / \mathrm{min} / 1.73$ $\mathrm{m}^{2}$. Postimplementation interviews took place with nine patients discharged from the CKD multidisciplinary clinic, the relative of one patient discharged from the clinic and 17 multidisciplinary CKD healthcare providers. Preimplementation and postimplementation of the new criteria, all patients from the CKD multidisciplinary and general nephrology clinics were invited to complete a paper-based experience survey, and all multidisciplinary CKD healthcare providers were asked to complete an online job satisfaction survey.

Che et $a l^{19}$ retrospectively compared the outcomes of 643 patients from the Multi-Care-Kidney-Clinic, for patients with advanced CKD, before and after the revision of the clinic eligibility criteria, in Ontario, Canada. The new criteria applied included eGFR $<15 \mathrm{~mL} / \mathrm{min} / 1.73$ $\mathrm{m}^{2}$ and KFRE 2-year risk $>10 \%$. If eligibility criteria were not met, patients were discharged from the clinic.

Lastly, Sendek et $a l^{21}$ applied the 4-variable KFRE (2-year risk $>15 \%$ ) to the population in Duke Connected Care, a Medicare Shared Savings Program that manages the healthcare of over 46000 Medicare patients. Patients alive and with no evidence of ESKD, a prior nephrology visit or acute kidney injury without chronic dysfunction were referred for 'population health rounding' where their electronic health record was reviewed weekly with a multidisciplinary team to decide on changes in management. Number of patients rounded each month, time per case and any actions taken were recorded.

Results are shown in table 2, along with key findings from the critical appraisal. The three themes identified are described further.

\section{Primary care to specialty care interface}

Two studies ${ }^{18} 20$ reported outcomes following the introduction of a new referral process incorporating a 5-year ESKD risk threshold of $>3 \%$ to triage patients from primary care to specialty care, although they also incorporated additional referral criteria for eligibility for specialty care review. Both studies measured the number of consults preimplementation and postimplementation of the KFRE: Hingwala et al found monthly referrals increased by $45 \%,{ }^{18}$ whereas Hong et al found referrals decreased by $25 \%-30 \%{ }^{20}$

Hingwala et al also showed a significant reduction in the wait-time from referral to review (median 58 vs 230 days) following implementation of the new triage system. ${ }^{18}$

\section{General nephrology to multidisciplinary/advanced kidney care clinic}

Patients under nephrology care may be managed in a general nephrology clinic or, for patients with more advanced CKD, a multidisciplinary clinic focused on managing CKD complications and RRT preparation. Two studies examined the use of the KFRE at this interface and introduced similar eligibility criteria for entry into and management in the multidisciplinary clinic: a 2-year ESKD risk $>10 \%$ or eGFR $<15 \mathrm{~mL} / \mathrm{min} / 1.73 \mathrm{~m}^{2}$ (formula used for calculating eGFR not specified in the studies).

Che $e t a l^{19}$ found that by applying these criteria to all prevalent patients in the multidisciplinary clinic, $73 \%$ remained in the clinic, $5 \%$ were stepped down to general nephrology care and 22\% were discharged to primary care. Of the latter, $11 \%$ required rereferral to nephrology, 

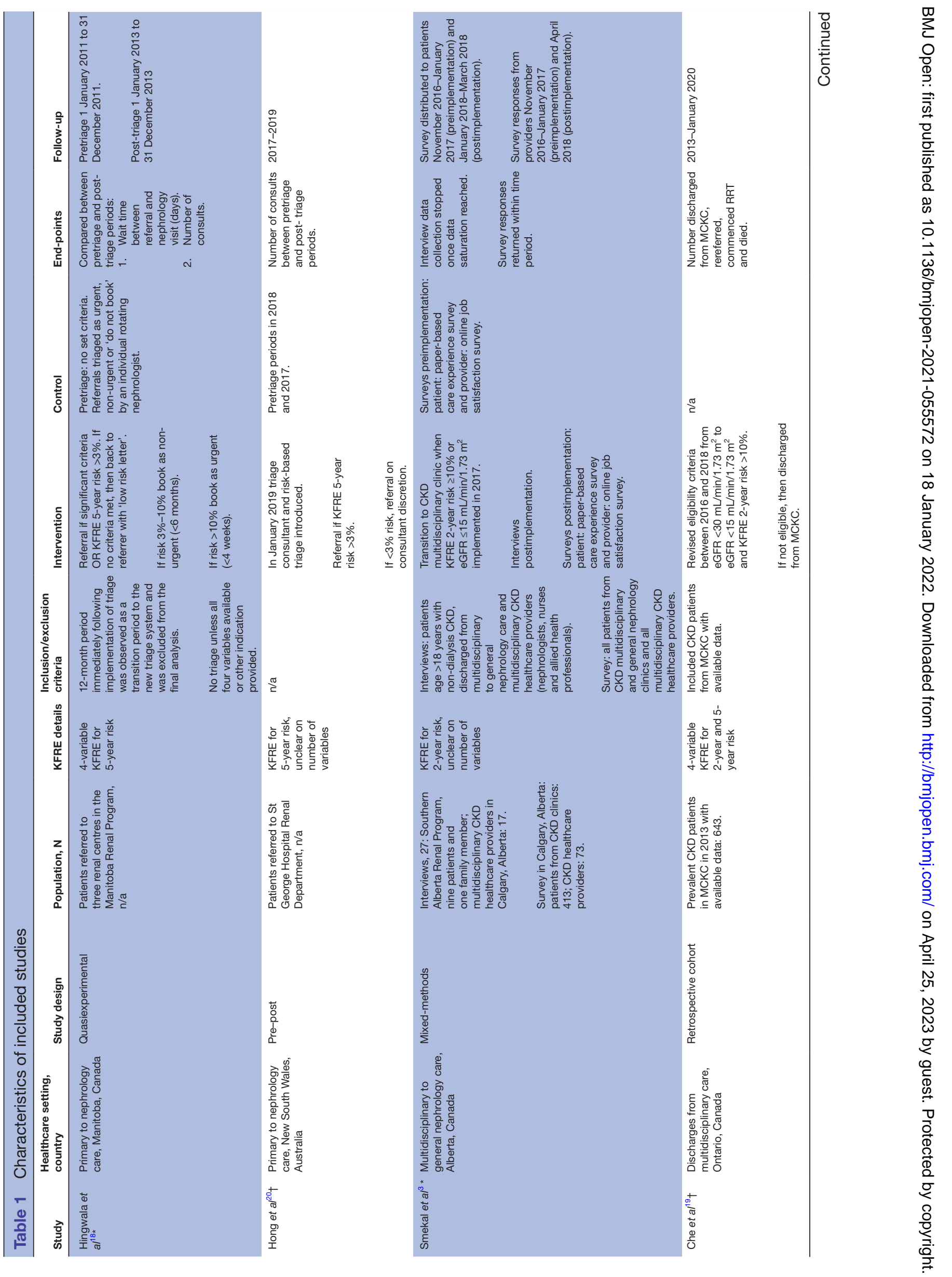


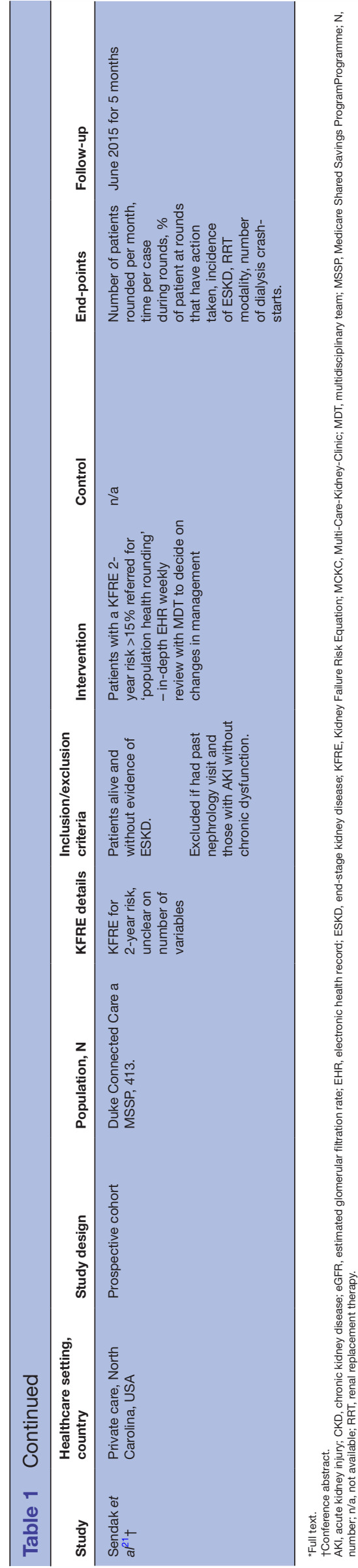

and $6 \%$ ultimately required RRT, although the majority in the context of an unforeseen acute illness.

Smekal $e t a l^{3}$ evaluated patient and healthcare providers' views and experiences following the implementation of the new KFRE-based criteria for multidisciplinary clinic management. Providers felt using KFRE to target highrisk patients was a key strength, limiting inappropriate referrals and improving the clinic's focus. The caseload was felt to be more 'acute' but overall workload not significantly changed, and there was no significant difference in job satisfaction. Providers also expressed concern that there may be inadequate access to and a lower quality of care for low-risk patients discharged from the multidisciplinary clinic, such as with education and monitoring. Some nurses reported discharged patients were contacting them for test results, and patients reported missing this aspect of their care. Although there was some improvement in patients' experience of access to care, caring staff and safety of care, most patients were satisfied with their care both preimplementation and postimplementation, and there was no difference in patients' overall care experience.

\section{Treatment planning}

The study by Sendak $e t a l^{21}$ took place in an 'Accountable Care Organisation' within the Medicare Shared Savings Program (Medicare is a health insurance programme mainly for people aged $\geq 65$ years in the USA). A 2-year ESKD risk threshold of $>15 \%$ was applied to the cohort to identify high-risk CKD patients, not already under nephrology review, for a multidisciplinary review of their electronic healthcare record (EHR). The reviews were able to be performed relatively rapidly (average $2 \min 12$ s) and led to changes in management in $21.8 \%$ of cases, most often a referral for nephrology review.

\section{Critical appraisal}

All 5 studies were limited to a single centre or region. No randomised control trials were identified. Two were cohort studies $^{1921}$, one was a mixed-method study ${ }^{3}$ and two were quasiexperiment studies. ${ }^{18}{ }^{20}$ All studies stated a clear aim or issue to be addressed. Sample size varied depending on the study method, and two studies did not state it. ${ }^{1820}$ Participant baseline characteristics data were not available in two studies ${ }^{19} 20$ and lacking in one study ${ }^{18}$ where preintervention information was not available. For the qualitative aspect of the mixed-methods study, ${ }^{3}$ the patients selected were English-speaking only. Although this may have allowed for simpler data analysis as there was no requirement for language translation, this selection bias may limit the perspectives obtained of those experiencing the intervention. In this same study, the survey response rate for patients was unclear, and for providers, no data were provided to establish any difference in demographics to non-responders. Although mostly proportions were reported in the data analysis of quantitative studies, there was a lack of reporting of statistical significance, strength of any associations or measures 


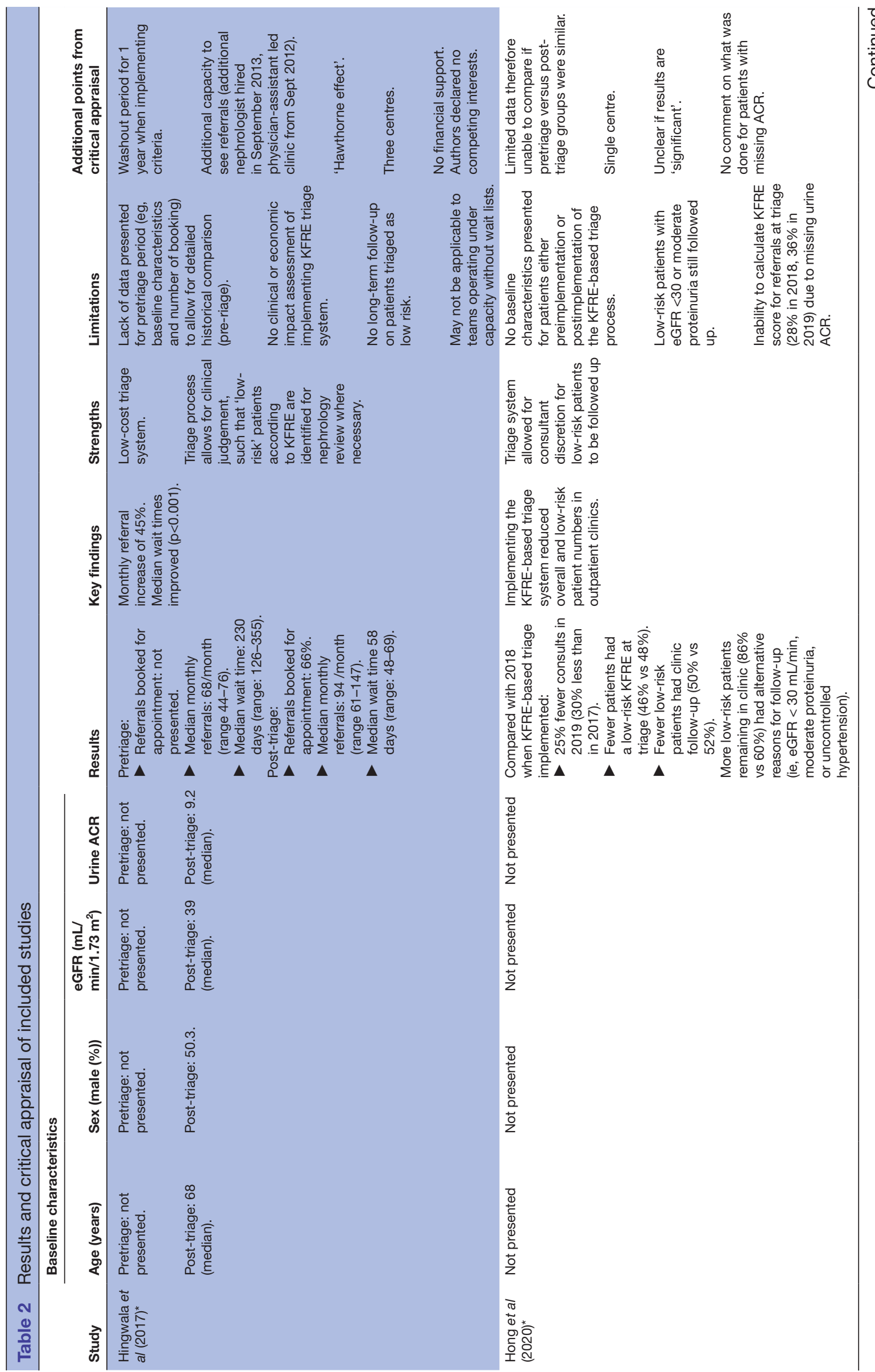




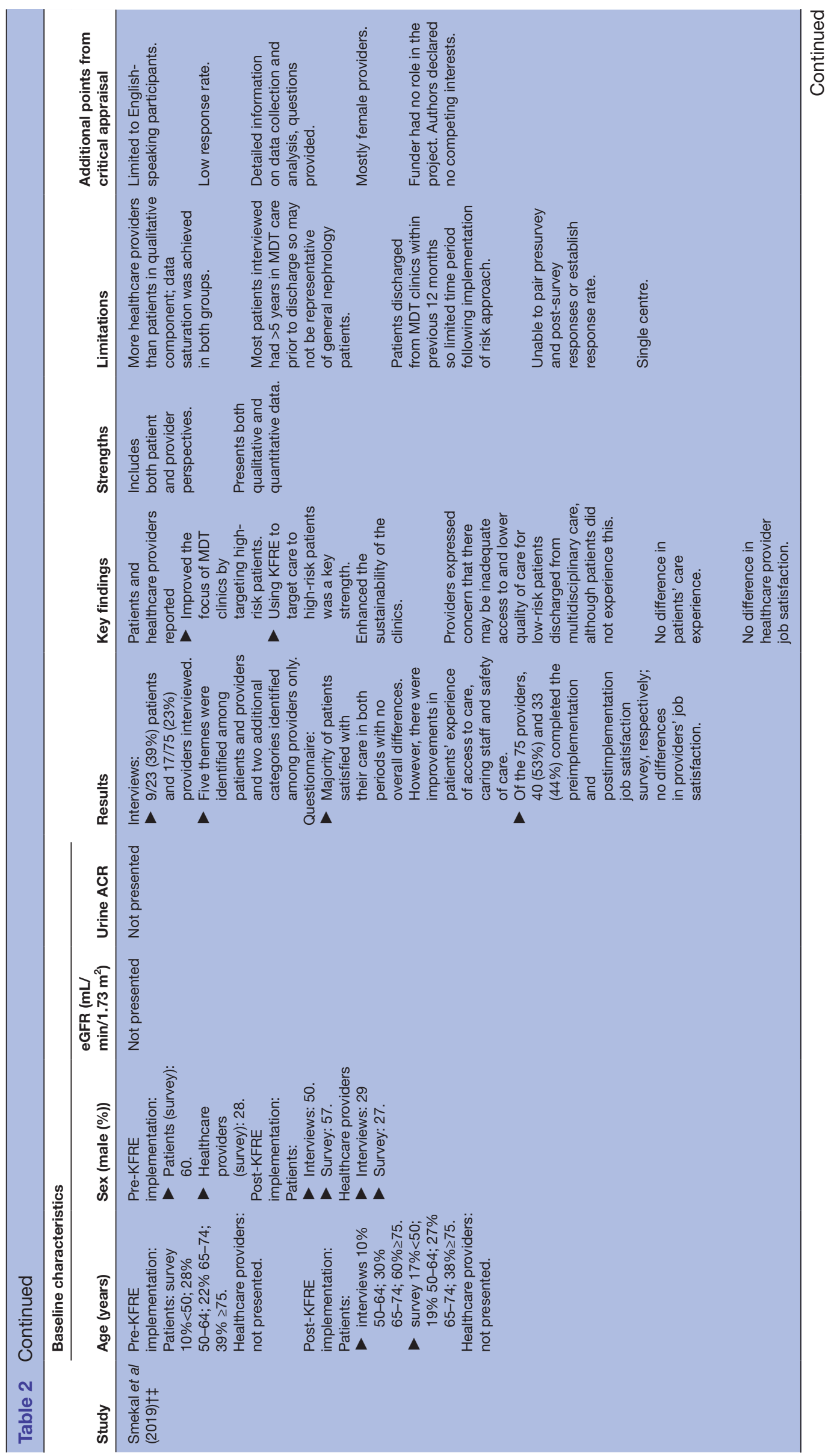




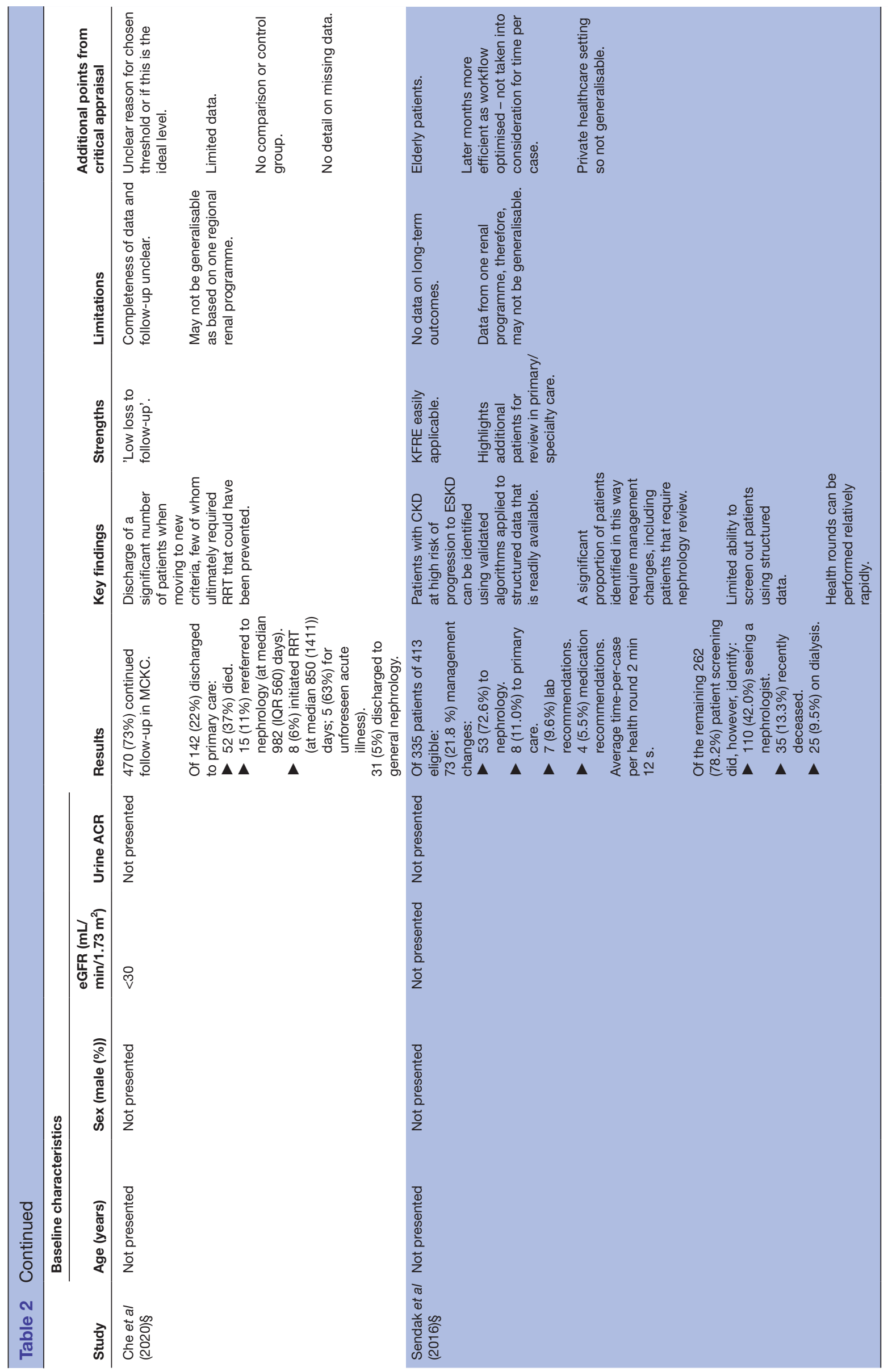




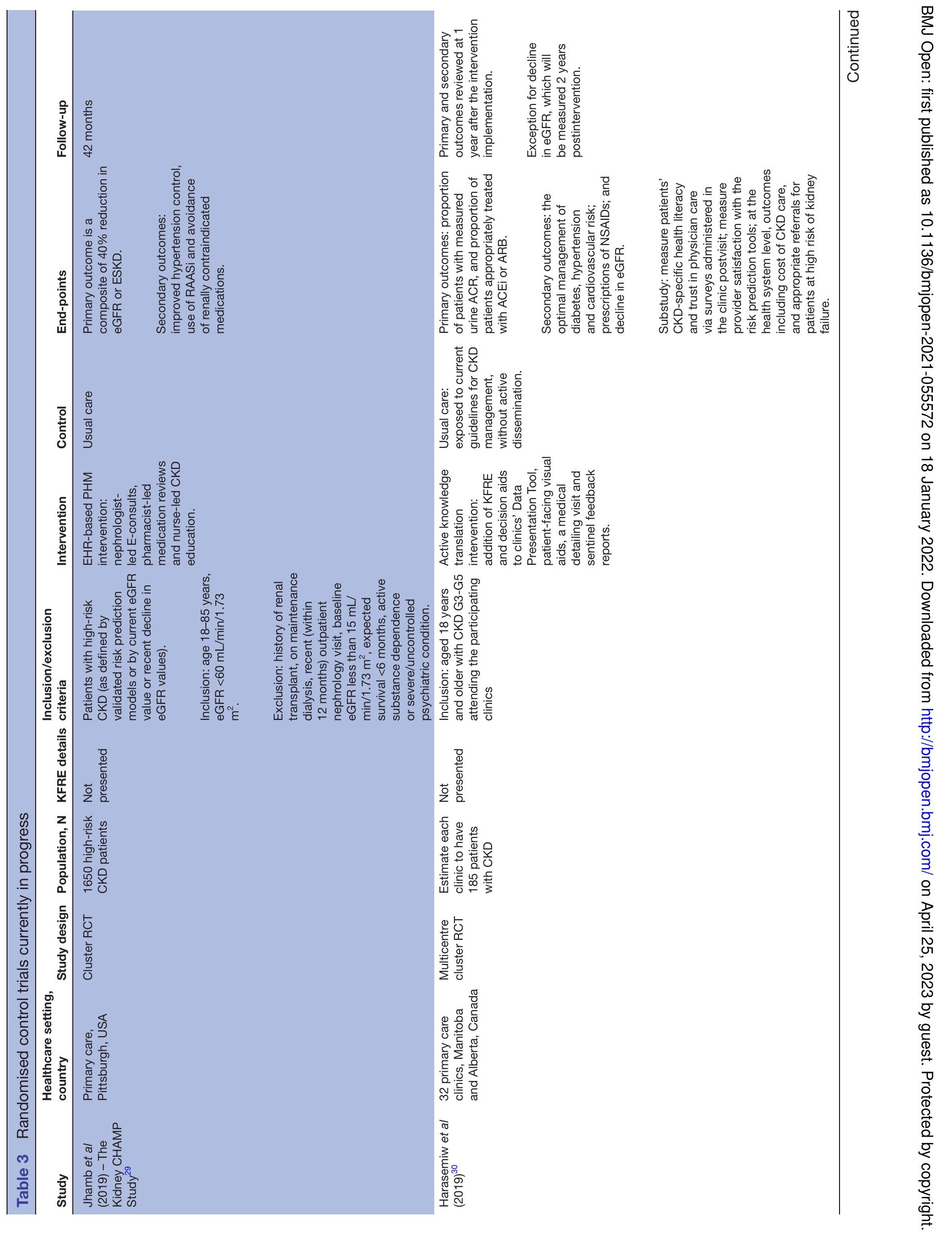




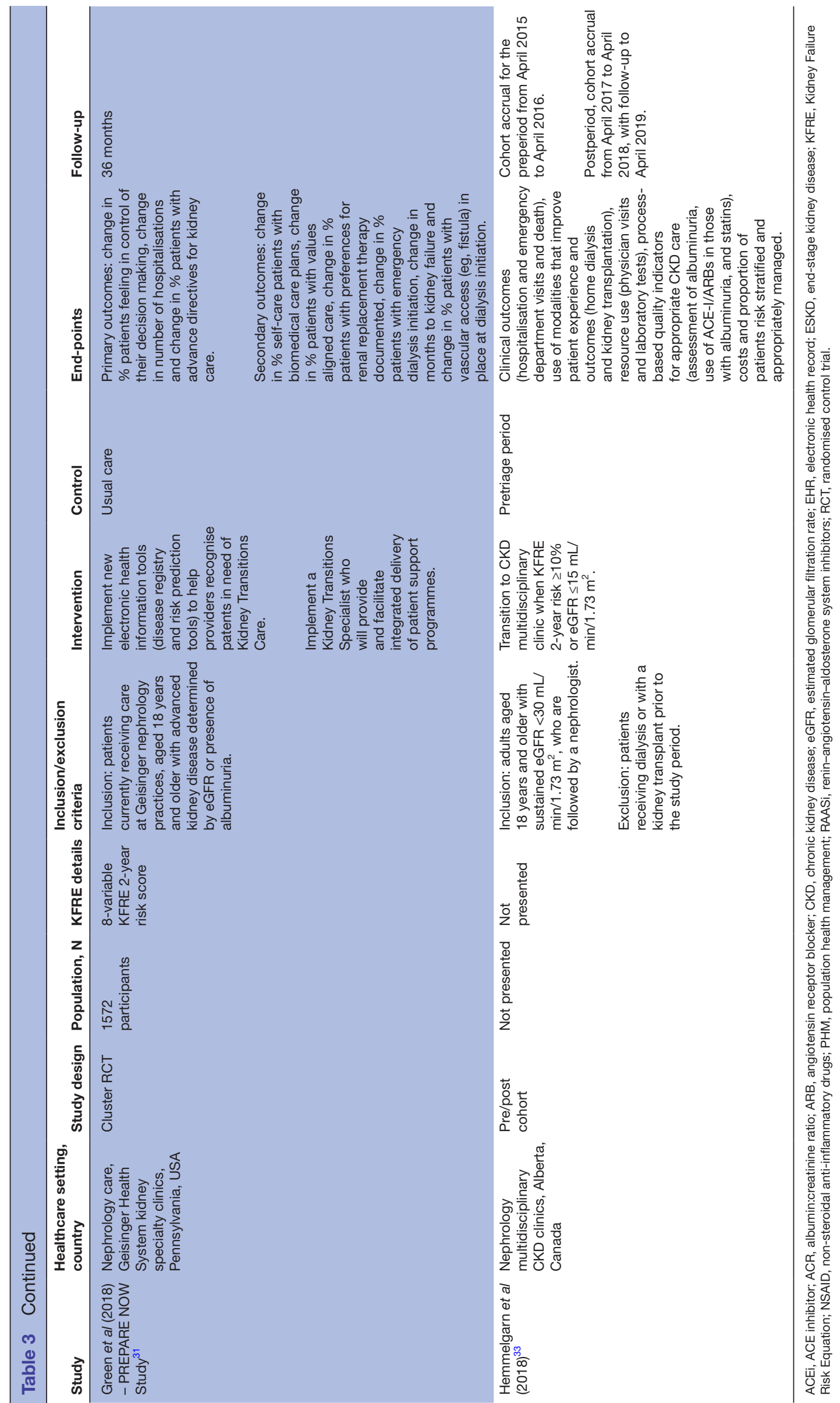


of precision in most. Three studies ${ }^{19-21}$ reported only proportions when applying the intervention. These three studies had not commented on the number of patients, if any, who could not be assessed for risk due to missing data or whether there were any confounding factors.

\section{DISCUSSION}

In this systematic review, we identified five studies of varying methodologies evaluating the actual impact of utilising the KFRE in clinical practice for patients with CKD. The equation was used in three main areas: (1) triaging patients between primary and specialty nephrology care, (2) directing patients between general nephrology and multidisciplinary advanced CKD care clinics; and (3) treatment planning where high-risk patients within a healthcare programme were identified for a multidisciplinary EHR review.

\section{Interpretation of the results in the context of other evidence}

The findings of previous studies exploring the potential impact of applying the KFRE at the primary care speciality care interface suggest referral numbers could potentialy increase depending on the threshold criteria set. ${ }^{522} 23$ This is consistent with the findings of Hingwala $e t$ $a l,{ }^{18}$ who reported an increase in referrals. However, wait times improved, possibly related to additional capacity to see referrals and a 'Hawthorne effect' (better performance as a result of healthcare professionals' awareness of being observed in a study). ${ }^{24}$ In contrast, Hong et al, ${ }^{20}$ who implemented the same risk threshold, reported a fall in referrals. These contrasting findings likely reflect differences in local practice and case-mix, whereby a higher proportion of low-risk patients (ie, older, higher eGFRs, lower urine ACRs) were managed by nephrology rather than by primary care before implementation of the KFRE criteria, compared with the centre reported by Hingwala $e t a l$. We were unable to review any population differences pretriage and post-triage or between studies due to a lack of reported data. ${ }^{18} 20$

In specialist nephrology care, many centres have advanced kidney care clinics for those patients who require increased multidisciplinary support to manage CKD complications and prepare for RRT. ${ }^{25}{ }^{26}$ The KFRE has the potential to identify patients at higher risk of ESKD to more efficiently direct increased support and resources for this group. Those identified as low risk can be stepped down, reducing clinic burden and unnecessary interventions. Two studies evaluated this part of the CKD pathway and applied similar eligibility criteria for multidisciplinary clinic management. Che $e t a l^{19}$ found many patients could be discharged from the multidisciplinary clinic, the majority back to primary care, with only a small number referred back to nephrology care or ultimately requiring RRT.

The findings of Smekal et a $\vec{l}$ suggest some anxiety regarding reduced access to services, education and monitoring when patients are discharged from the multidisciplinary clinic to general nephrology care based on their KFRE-calculated risk. Maintaining patient and provider satisfaction with CKD care when using the KFRE to discharge patients is an important issue that will require the considered configuration of local renal service delivery to ensure accessibility and patient safety. It remains unknown if the application of the KFRE to identify higher risk patients for multidisciplinary care impacts key outcomes such as progression of CKD, commencing RRT, cardiovascular events or mortality.

Sendak $e t a l^{21}$ reviewed the impact of using KFRE in a private healthcare setting in a primarily elderly population to identify patients who required input from medical teams (in primary or specialist care), treatment changes or additional lab testing. The results of this study may not be easily generalisable as this is not a widely used provider model in some countries.

Other suggested areas of use have been for planning RRT in an elderly population where competing risk of death is of concern ${ }^{27}$ and for creation of vascular access, ${ }^{28}$ but no studies have implemented and evaluated this.

\section{Limitations of the evidence included in the review and the review process}

The studies varied widely in methodologies used. The benefit of this mixed-methods review is a greater breadth of understanding and evidence around the application of the risk-based KFRE in clinical practice. This can provide a more rounded body of evidence to inform changes in clinical practice and policy decision making. No randomised control trials were identified.

Study quality also ranged widely. Some studies did not provide adequate detail of their population characteristics. As a result, it was difficult to assess if the population was representative or findings could be generalised. Recognising differences between study populations was also limited by the lack of data. Few studies adequately completed statistical analysis and so it was challenging to decipher significant findings. Authors of the identified studies were not approached for additional information, and we recognise this as a limitation.

\section{Implication of the results for practice, policy and future research}

While this study has identified the ease of use of the KFRE to triage patients and highlight those who would benefit from changes in management, plus the potential effect on the number of referrals and patients' and providers' experience and perspectives, the impact on health outcomes and economic benefit are still unknown.

More high-quality studies are needed to confidently support the widespread use of KFRE, particularly randomised control trials with a focus on health outcomes and economic impact. Four trials that are in currently progress will assess the outcomes of applying the KFRE (table 3). The Kidney CHAMP study, ${ }^{29}$ a cluster randomised controlled trial in Pittsburgh, USA, will review the effectiveness of integrating the KFRE 
into EHR to identify patients at high risk of progression who require intervention such as nephrology guidance, medication reviews and targeted CKD education. Outcomes assessed will be a composite of a $40 \%$ reduction in eGFR and ESKD. A multicentre cluster randomised control trial in Canada ${ }^{30}$ aims to review the risk-based approach in the primary care setting. The intervention, providing patients and providers in primary care with a patient's KFRE score and the riskbased referral criteria, will be reviewed to assess if it improves appropriate patient management, improves a patient's CKD-specific health literacy and affects the cost of care compared with usual care. The PREPARE NOW study, ${ }^{31}$ a cluster randomised control trial within a nephrology specialty care setting in Pennsylvania, USA, is applying the KFRE among a suite of digital tools integrated with EHRs to alert the healthcare team of patients at risk of progression and need for intervention. This will be in addition to multiple other components, and patient-reported, biomedical and health system outcomes will be collected. Early findings support the 'ease of use' and 'helpfulness' of the tools. ${ }^{32}$ The fourth trial, with details described in the protocol by Hemmelgarn et $a l^{33}$ is a multiphase mixedmethods study. Following the completion of phase 2, findings have been published by Smekal et al, ${ }^{3}$ a study that met the criteria for inclusion in this systematic review. The whole trial took on a pre-post design and applied the KFRE to patients in nephrology multidisciplinary CKD clinics in Alberta, Canada. The final phase will aim to evaluate the costs of care and outcomes before and after the introduction of risk-based triage, such as healthcare resource use, frequency of testing, modality choice and death.

Despite the lack of sufficient impact studies, strong evidence to date from validation studies and studies investigating potential impact in clinical practice have been encouraging. As a result, several CKD guidelines have or are in the process of incorporating KFRE risk-based criteria in their pathways. ${ }^{6734}$

\section{CONCLUSION}

The KFRE has been extensively validated, but there has been relatively little evaluation of its clinical and economic impact. This is the first study to systematically review studies exploring at the actual impact of using the KFRE in clinical practice. Additional high-quality studies are required, and trials assessing the impact of using KFRE at various stages across the CKD pathway are in progress.

\footnotetext{
Author affiliations

${ }^{1}$ Department of Renal Medicine, University Hospitals Birmingham NHS Foundation Trust, Birmingham, UK

${ }^{2}$ Institute of Applied Health Research, University of Birmingham, Birmingham, UK ${ }^{3}$ Centre for Patient-Reported Outcomes Research (CPROR), Institute of Applied Health Research, University of Birmingham, Birmingham, UK

${ }^{4}$ Institute of Inflammation and Ageing, University of Birmingham, Birmingham, UK
}

${ }^{5}$ NIHR Birmingham Biomedical Research Centre, NIHR Surgical Reconstruction and Microbiology Research Centre and NIHR ARC, Birmingham, UK

${ }^{6}$ Birmingham Health Partners Centre for Regulatory Science and Innovation, University of Birmingham, Birmingham, UK

${ }^{7}$ School of Allied Health and Community, University of Worcester, Worcester, UK

Contributors HKB, PC, MC and DK conceived the study. HKB developed the methodology with guidance from PC, MC, DK, OA and AF. The database, reference and citation searches were completed by HKB and AF. Screening and selection of the included studies was conducted by HKB and AF; PC as the third reviewer was consulted for any disagreements. HKB and AF critically appraised the included studies. HKB drafted the initial manuscript and all authors contributed to revision and approval of the final manuscript. HKB, as guarantor, accepts full overall responsibility for the conduct of this study.

Funding This project received funding from University Hospitals Birmingham Charity (charity register number: 1165716) - Renal Research Fund (no award/grant number).

Competing interests PC reports grants from Kidney Research UK, National Institute for Health Research (NIHR), MRC and EU Horizon 20/20 programme, and personal fees from NAPP Pharmaceuticals outside the submitted work. $O A$ receives funding from the NIHR Birmingham Biomedical Research Centre, NIHR Applied Research Centre (ARC), West Midlands at the University of Birmingham and University Hospitals Birmingham NHS Foundation, Innovate UK (part of UK Research and Innovation), Gilead Sciences Ltd and Janssen Pharmaceuticals, Inc. OLA declares personal fees from Gilead Sciences Ltd, GlaxoSmithKline (GSK) and Merck outside the submitted work. DK reports grants from Macmillan Cancer Support, Innovate UK, the NIHR, NIHR Birmingham Biomedical Research Centre and NIHR SRMRC at the University of Birmingham and University Hospitals Birmingham NHS Foundation Trust, and personal fees from Merck and GSK outside the submitted work. MC is an NIHR Senior Investigator and receives funding from the NIHR Birmingham Biomedical Research Centre, the NIHR Surgical Reconstruction and Microbiology Research Centre and NIHR ARC West Midlands at the University of Birmingham and University Hospitals Birmingham NHS Foundation Trust, Health Data Research UK, Innovate UK (part of UK Research and Innovation), Macmillan Cancer Support, UCB Pharma and GSK. MC has received personal fees from Astellas, Takeda, Merck, Daiichi Sankyo, Glaukos, GSK and the Patient-Centered Outcomes Research Institute outside the submitted work.

Patient consent for publication Not applicable.

Ethics approval This study does not involve human participants.

Provenance and peer review Not commissioned; externally peer reviewed.

Data availability statement Data sharing not applicable as no datasets generated and/or analysed for this study. Data sharing not applicable for this systematic review.

Supplemental material This content has been supplied by the author(s). It has not been vetted by BMJ Publishing Group Limited (BMJ) and may not have been peer-reviewed. Any opinions or recommendations discussed are solely those of the author(s) and are not endorsed by BMJ. BMJ disclaims all liability and responsibility arising from any reliance placed on the content. Where the content includes any translated material, BMJ does not warrant the accuracy and reliability of the translations (including but not limited to local regulations, clinical guidelines, terminology, drug names and drug dosages), and is not responsible for any error and/or omissions arising from translation and adaptation or otherwise.

Open access This is an open access article distributed in accordance with the Creative Commons Attribution Non Commercial (CC BY-NC 4.0) license, which permits others to distribute, remix, adapt, build upon this work non-commercially, and license their derivative works on different terms, provided the original work is properly cited, appropriate credit is given, any changes made indicated, and the use is non-commercial. See: http://creativecommons.org/licenses/by-nc/4.0/.

\section{ORCID iDs}

Anthony Fenton http://orcid.org/0000-0003-2665-6924

Derek Kyte http://orcid.org/0000-0002-7679-6741

Melanie Calvert http://orcid.org/0000-0002-1856-837X

\section{REFERENCES}

1 Bikbov B, Purcell CA, Levey AS, et al. Global, regional, and national burden of chronic kidney disease, 1990-2017: a systematic 
analysis for the global burden of disease study 2017. Lancet 2020;395:709-33.

2 Daker-White G, Rogers A, Kennedy A, et al. Non-disclosure of chronic kidney disease in primary care and the limits of instrumental rationality in chronic illness self-management. Soc Sci Med 2015;131:31-9.

3 Smekal MD, Tam-Tham H, Finlay J, et al. Patient and provider experience and perspectives of a risk-based approach to multidisciplinary chronic kidney disease care: a mixed methods study. BMC Nephrol 2019;20.

4 National Institute for Health and Care Excellence (UK). Chronic kidney disease in adults: assessment and management (CG182). London: National Institute for Health and Care Excellence (UK), 2014.

5 Bhachu HK, Cockwell P, Subramanian A, et al. Impact of using Risk-Based stratification on referral of patients with chronic kidney disease from primary care to specialist care in the United Kingdom. Kidney Int Rep 2021;6:2189-99.

6 Kidney disease: improving global outcomes (KDIGO) CKD work group. KDIGO 2012 clinical practice guideline for the evaluation and management of chronic kidney disease. Kidney Inter supp/ 2013:3:1-150.

7 Farrington K, Covic A, Aucella F. Clinical practice guideline on management of older patients with chronic kidney disease stage 3B or higher (eGFR $<45 \mathrm{~mL} / \mathrm{min} / 1.73 \mathrm{~m}^{2}$ ). Nephrology Dialysis Transplantation 2016;31:ii1-66.

8 Ramspek CL, de Jong Y, Dekker FW, et al. Towards the best kidney failure prediction tool: a systematic review and selection aid. Nephrol Dial Transplant 2020;35:1527-38.

9 Tangri N, Grams ME, Levey AS, et al. Multinational assessment of accuracy of equations for predicting risk of kidney failure: a metaanalysis. JAMA 2016;315:164-74.

10 Ramspek C, de Jong Y, Dekker F, et al. FP367PREDICTIVE models for progression of chronic kidney disease to kidney failure: a systematic review. Nephrology Dialysis Transplantation 2018;33:1155.

11 The kidney failure risk equation.

12 Liberati A, Altman DG, Tetzlaff $\mathrm{J}$, et al. The PRISMA statement for reporting systematic reviews and meta-analyses of studies that evaluate health care interventions: explanation and elaboration. PLOS Med 2009;6:e1000100.

13 Tangri N, Stevens LA, Griffith J, et al. A predictive model for progression of chronic kidney disease to kidney failure. JAMA 2011;305:1553-9.

14 Critical appraisal skills programme. Available: https://casp-uk.net/ [Accessed 14 Apr 2021].

15 Joanna Briggs Institute critical appraisal tools. Available: https://jbi. global/critical-appraisal-tools [Accessed 14 Apr 2021].

16 Center for Evidence-Based Management (CEBMa). Critical appraisal of a survey. Available: https://www.cebma.org/wp-content/uploads/ Critical-Appraisal-Questions-for-a-Survey.pdf [Accessed $14 \mathrm{Apr}$ 2021].

17 Popay J, Roberts H, Sowden A. Guidance on the conduct of narrative synthesis in systematic reviews: a product from the ESRC methods programme 2006

18 Hingwala J, Wojciechowski P, Hiebert B, et al. Risk-Based triage for nephrology referrals using the kidney failure risk equation. Can $J$ Kidney Health Dis 2017;4:205435811772278.
19 Che M, Thanabalasingam SJ, lliescu EA. Patient outcomes following discharge from a CKD clinic. Journal of the American Society of Nephrology 2020;31:227.

20 Hong R, Pirabhahar S, Turner K. Triage system for nephrology referrals using the kidney failure risk equation (KFRE) score. Nephrology 2020;25:53.

21 Sendak M, Cameron B, Komives E. Developing a data-driven work flow for population health rounding. Journal of General Internal Medicine 2016;31:S874-5.

22 Slattery LM. Applying novel kidney failure risk equations applications to new patient referrals: a 6-month retrospective review. Journal of the American Society of Nephrology 2019;30:1173.

23 Major RW, Shepherd D, Medcalf JF, et al. The kidney failure risk equation for prediction of end stage renal disease in UK primary care: an external validation and clinical impact projection cohort study. PLoS Med 2019;16:e1002955.

24 McCambridge J, Witton J, Elbourne DR. Systematic review of the Hawthorne effect: new concepts are needed to study research participation effects. J Clin Epidemiol 2014;67:267-77.

25 Nicoll R, Robertson L, Gemmell E, et al. Models of care for chronic kidney disease: a systematic review. Nephrology 2018;23:389-96.

26 Evans M, Lopau K. The transition clinic in chronic kidney disease care. Nephrol Dial Transplant 2020;35:ii4-10.

27 Nastasa A, Apetrii M, Onofriescu M, et al. P0184RISK prediction for death and end-stage renal disease does not parallel real-life trajectory of older patients with advanced chronic kidney disease - a romanian center experience. Nephrology Dialysis Transplantation 2020;35:iii486.

28 Inston N, Lok CE. Improving precision in prediction: using kidney failure risk equations as a potential adjunct to vascular access planning. J Vasc Access 2019;20:95-7.

29 Jhamb M, Yabes J, Fischer G. Electronic health record based population health management for improving CKD care: the kidneycoordinated health management partnership (Champ) study. JASN 2019;30:525.

30 Harasemiw O, Drummond N, Singer A, et al. Integrating Risk-Based care for patients with chronic kidney disease in the community: study protocol for a cluster randomized trial. Can J Kidney Health Dis 2019;6:205435811984161.

31 Green JA, Ephraim PL, Hill-Briggs FF, et al. Putting patients at the center of kidney care transitions: prepare now, a cluster randomized controlled trial. Contemp Clin Trials 2018;73:98-110.

32 Green JA, Ephraim PL, Hill-Briggs F, et al. Integrated digital health system tools to support decision making and treatment preparation in CKD: the prepare now study. Kidney Med 2021;3:565-75

33 Hemmelgarn BR, Smekal MD, Weaver RG, et al. Implementation and evaluation of a risk-based approach to guide chronic kidney disease care: protocol for a multiphase mixed-methods study. Can J Kidney Health Dis 2018;5:205435811775361.

34 National Institute for Health and Care Excellence (UK). Chronic kidney disease: assessment and management (NG203). London: National Institute for Health and Care Excellence (UK) 2021. 